\title{
Biocompatible Magnetite Nanoparticles Trapped at the Air/Water Interface
}

\author{
Cristina Stefaniu, ${ }^{*[a]}$ Munish Chanana ${ }^{[a]}$ Dayang Wang, ${ }^{[a]}$ Dmitri V. Novikov, ${ }^{[b]}$ Gerald Brezesinski, ${ }^{*[a]}$ and \\ Helmuth Möhwald ${ }^{[a]}$
}

In the last decade, iron oxide nanoparticles (NPs) — known as super-paramagnetic iron oxide nanoparticles (SPION) - have received significant attention due to their applicability in a variety of domains. In the biomedical field, ${ }^{[1]}$ iron oxide nanoparticles are very promising as drug-delivery systems, ${ }^{[2-4]}$ magneticresonance-imaging contrast enhancers (clinical diagnosis), ${ }^{[5-6]}$ inflammation-responsive or anti-cancer agents, or for labeling and cell separation. In addition to their potential medical applications, magnetic nanoparticles are of great interest in materials science, for the development of magnetic data-recording media and nanocomposite permanent magnets, ${ }^{[7]}$ as well as in catalysis and colloid chemistry, where ferrofluids ${ }^{[8-9]}$ represent a topic of high interest.

This study is focused on $\mathrm{Fe}_{3} \mathrm{O}_{4}$ NPs grafted with the thermosensitive and biocompatible copolymer $\mathrm{MEO}_{2} \mathrm{MA} / \mathrm{OEGMA}{ }^{[10]}$ whose interfacial behavior is yet unknown. Many papers have been dedicated to the formation, ${ }^{[11-12],}$ organization, ${ }^{[13]}$ and stability ${ }^{[14-15]}$ of Langmuir films of iron oxide $\left(\mathrm{Fe}_{2} \mathrm{O}_{3}\right.$ or $\left.\mathrm{Fe}_{3} \mathrm{O}_{4}\right) \mathrm{NPs}$ and their Langmuir-Blodgett transfer. ${ }^{[16]}$ The system studied herein is completely different from the iron oxide NPs already studied at the air/water interface due to the unique properties of the polymer used. A monodisperse population of uncharged $\mathrm{Fe}_{3} \mathrm{O}_{4}$ cores with a diameter of $6.4 \mathrm{~nm}$, which are grafted with catechol-terminated copolymers of 2-(2-methoxyethoxy) ethyl methacrylate (MEO2MA) and oligo(ethylene glycol) methacrylate (OEGMA), ${ }^{[10]}$ has been investigated (Figure 1). Due to the presence of oligo(ethylene glycol) side groups on the sur-

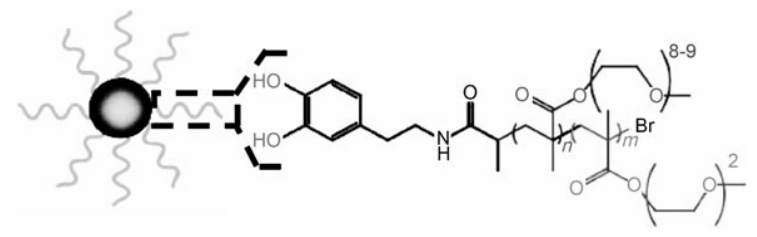

Figure 1. Schematic representation of the $\mathrm{Fe}_{3} \mathrm{O}_{4} @ \mathrm{MEO}_{2} \mathrm{MA}_{90}$-Co-OEGMA NPs

[a] Dr. C. Stefaniu, Dr. M. Chanana, Dr. D. Wang, Prof. Dr. G. Brezesinski, Prof. Dr. H. Möhwald

Max Planck Institute of Colloids and Interfaces

14476 Potsdam (Germany)

Fax: (+ 49) 331-567-9234

E-mail:brezesinski@mpikg.mpg.de stefaniu@mpikg.mpg.de

[b] Dr. D. V. Novikov

HASYLAB, DESY, Notkestraße 85

22607 Hamburg (Germany)

Supporting information for this article is available on the WWW under http://dx.doi.org/10.1002/cphc.201000783. faces, the $\mathrm{Fe}_{3} \mathrm{O}_{4} @ \mathrm{MEO}_{2} \mathrm{MA}_{90}$-CO-OEGMA 10 NPs (90 and 10 represent the molar fractions of $\mathrm{MEO}_{2} \mathrm{MA}$ and OEGMA, respectively) can be dispersed in water, exhibiting a high colloidal stability against salt and, at the same time, they can be well dispersed in organic solvents such as chloroform, ethanol or toluene. This specific copolymer in water (no salt) is hydrophilic and becomes hydrophobic at temperatures above $40^{\circ} \mathrm{C} .^{[10]}$ The experimental details are presented in the Supporting Information.

Since the polymer and the polymer-dressed NPs behave exactly in the same way, showing that the polymer dictates the surface activity, we will describe here mainly the results obtained with the polymer-dressed NPs because this system can be investigated with a larger number of methods giving complementary information. The surface activity of the $\mathrm{Fe}_{3} \mathrm{O}_{4} @ \mathrm{MEO}_{2} \mathrm{MA}_{90}-\mathrm{Co}-\mathrm{OEGMA}{ }_{10} \mathrm{NPs}$ is based on the amphiphilic character of the copolymer shell. This (oligo ethylene glycol) methyl ether methacrylate polymer has a graft structure (Figure 1) composed of an apolar carbon-carbon backbone which leads to a competitive hydrophobic effect and multiple oligo(ethylene glycol) side chains of which ether oxygen atoms form stabilizing hydrogen bonds with water. ${ }^{[17]}$ Moreover, the ethylene oxide motif can adopt a configuration with the oxygen atoms on one side of the molecule and with the two methylene groups on the other, thus giving the molecule both a hydrophilic and a hydrophobic surface. ${ }^{[18]}$ The hydrophobicity can be tuned by changing the molar fraction of the two monomers. ${ }^{[17]}$ The polymer can be dispersed both in water and in chloroform; therefore, the corresponding films have been prepared either by adsorption from aqueous bulk solution or by spreading from a chloroform solution at the interface. Using, for example, a NP bulk concentration of $1.5 \times 10^{-3} \mathrm{mg} \mathrm{mL}^{-1}$, a constant surface pressure value of approximately $23 \mathrm{mN} \mathrm{m}^{-1}$ is reached after $20 \mathrm{~h}$ (Figure $2 \mathrm{~A}$ ). Compression isotherms of Langmuir layers formed by spreading certain amounts of NPs are shown in Figure $2 \mathrm{~B}$. By compression, the surface pressure increases continuously to $25 \mathrm{mN} \mathrm{m}^{-1}$ (critical pressure $\pi_{c}$ of the polymer as well as the NP film). During further compression, a plateau region appears at which the surface pressure increases only slightly up to a maximum value of $27 \mathrm{mNm}^{-1}$. It is very important to highlight that no hysteresis of the compression/ expansion isotherms is observed when the interfacial film is compressed to surface pressures below the critical pressure of the Langmuir layer (Figure $2 \mathrm{C}$ ), suggesting that no loss of material from the interface occurs.

Based on this observation, we can calculate the interfacial concentration of NPs corresponding to the critical pressure. The critical concentration amounts to $(7.7 \pm 0.6) \times$ $10^{-4} \mathrm{mg} \mathrm{cm}^{-2}$. This value is in good agreement with the NP interfacial concentration of $8.2 \times 10^{-4} \mathrm{mg} \mathrm{cm}^{-2}$, which can be cal- 

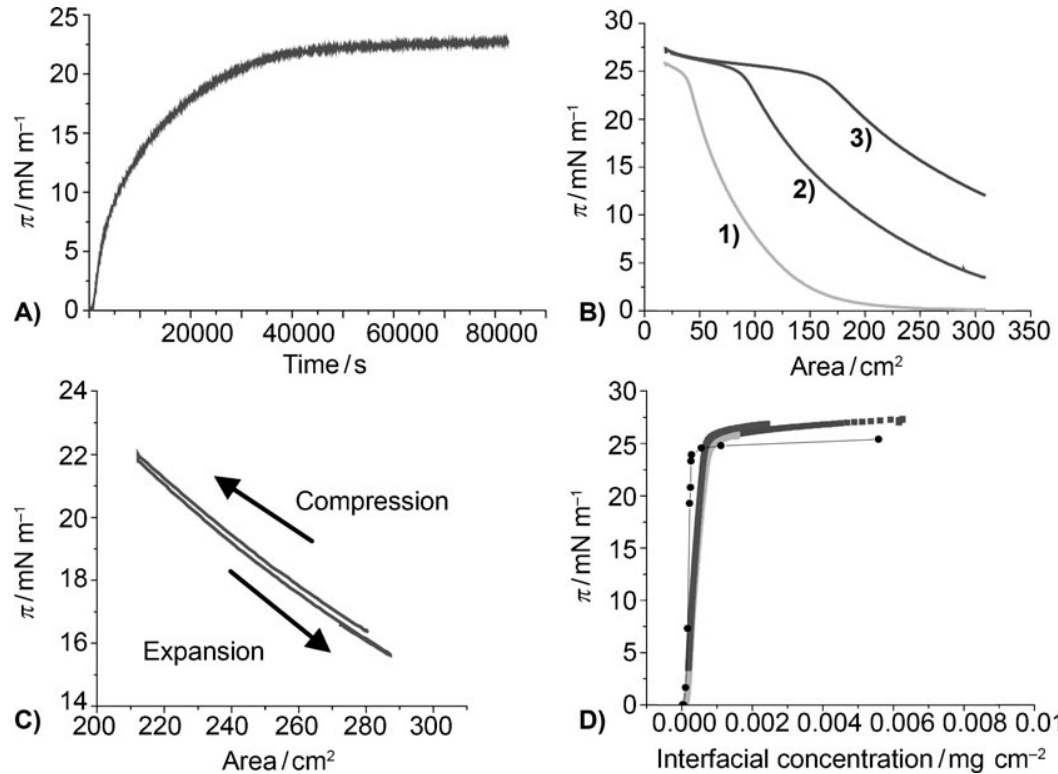

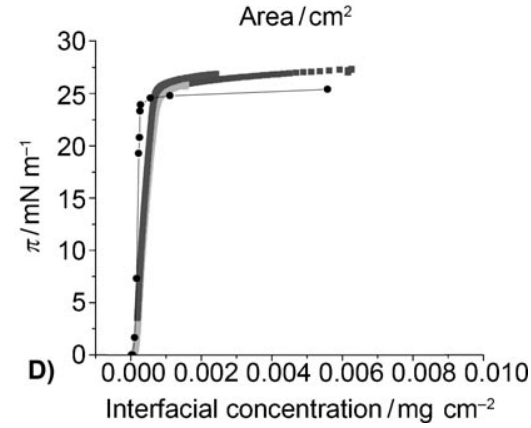

Figure 2. A) Adsorption of $\mathrm{Fe}_{3} \mathrm{O}_{4} \mathrm{NPs}$ at the surface of the aqueous bulk solution $\left(c=1.5 \times 10^{-3} \mathrm{mg} \mathrm{mL}^{-1}\right)$. B) Compression isotherms of three NP layers prepared by spreading [starting coverage: $9.74 \times 10^{-5}(1), 2.011 \times 10^{-4}(2)$, and $3.89 \times 10^{-4} \mathrm{mg} \mathrm{cm}^{-2}$ (3)] on Milli-Q Millipore water at $20^{\circ} \mathrm{C}$. C) Compression of the NP film (prepared by spreading) to only $22 \mathrm{mN} \mathrm{m}^{-1}$ and the following expansion after keeping the pressure constant for $80 \mathrm{~min}$. D) Variation of the surface pressure with the interfacial concentration of the NPs [circles (๑): PAT data; lines ( $\longrightarrow$ ): data from the compression isotherms presented in Figure 2B].

culated considering that all the NPs of the $1.5 \times 10^{-3} \mathrm{mg} \mathrm{mL}^{-1}$ aqueous solution adsorb at the air/water interface. The important conclusion is that the polymer and the corresponding NPs, once adsorbed at the interface, are trapped and do not exchange with material in the bulk.

A detailed study of the concentration-dependent adsorption behavior of the $\mathrm{Fe}_{3} \mathrm{O}_{4} @ \mathrm{MEO}_{2} \mathrm{MA}_{90}$-Co-OEGMA 10 NPs from aqueous solutions shows that the interfacial tension decreases with time and approaches an equilibrium value, which decreases with increasing concentration of the NPs. Above a certain concentration, which depends on the size of the air/water interface, a final surface tension of $47 \mathrm{mN} \mathrm{m}^{-1}$ is established. Using the same copolymer concentration, the minimum surface tensions are very similar for the two systems (copolymer-capped NPs and pure copolymer). This is a clear indication that the adsorption process is dictated by the copolymer, and the influence of the NPs is negligible. The lowest interfacial tension of $47 \mathrm{mN} \mathrm{m}^{-1}$ corresponds well to the critical pressure of the Langmuir monolayer showing that a maximum coverage (highest possible packing density) of the interface is attained.

Plotting the surface pressure versus the surface coverage of the interface shows that the result is absolutely independent of the way the surface coverage is obtained (either by spreading or by adsorption from the bulk solution) (Figure 2D). Previous studies have reported on identical compression isotherms of a polyvinylpyrrolidone-polyvinyl acetate graft copolymer obtained for spread and adsorbed films at the air/water interface. ${ }^{[19]}$ Some other studies showed that the structure of the adsorbed PEO monolayers is identical ${ }^{[20]}$ or very similar ${ }^{[21]}$ to that of the spread ones, without however quantifying and comparing the amount of the compound at the interface. The correspondence observed is an indication of the high flexibility of the fluid copolymer monolayers. This behavior is contrary to that of proteins which in most of the cases form thicker adsorbed films compared with the spread monolayers, explained by the retention of their native configuration at the surface when adsorbed, while spread protein molecules unfold more completely. ${ }^{[19]}$ However, it is noteworthy that protein layers with comparable characteristics were obtained for adsorbed and spread $\beta$-casein ${ }^{[22]}$ monolayers at oil/ water interfaces and for $\alpha$-lactalbumin $^{[23]}$ at the air/water interface. Therefore, one can say that our copolymer-NP system exhibits interfacial properties similar to a water soluble, conformational flexible protein.

Consequently, two more major observations need to be highlighted: Firstly, for the NP films formed by spreading, the NPs are trapped at the air/water interface and can be compressed until a critical pressure is reached. From this point of view, the NPs are similar to a water-insoluble amphiphilic compound. Secondly, all the NPs from the bulk adsorb at the interface, reaching a maximum coverage and the same critical equilibrium surface pressure as that observed for the Langmuir film. This behavior is completely different to that of a classical water-soluble surfactant for which (due to a free exchange) a partitioning between the interface and the bulk solution occurs.

At low concentrations, the amount of NPs is obviously not sufficient to reach a complete coverage of the interface. All the NPs adsorb at the interface to adopt an energetically favored state. At higher bulk concentrations, the critical packing density of the adsorption layer is reached and the remaining NPs have to stay in the aqueous bulk phase. This behavior can be explained by a conformational change of the copolymer chains from a brushlike structure, expressed in solution, to a pancake conformation at the air/water interface. A detailed study will be published elsewhere.

The compression/expansion $\pi / A$ isotherms of the adsorption layer and those of a Langmuir layer of NPs formed by spreading show very similar behaviors with a pronounced hysteresis (Figure $3 \mathrm{~B} 1$ ). To understand the NP loss in the plateau region above $\pi_{c}$ two scenarios are possible: i) Either an irreversible multilayer film is formed on top of the water surface, which does not re-spread after expansion, or ii) the NPs are squeezed out from the interface and re-dispersed into the aqueous bulk. In order to establish which scenario is valid, a dedicated and unique experiment has been performed: A layer of NPs was 

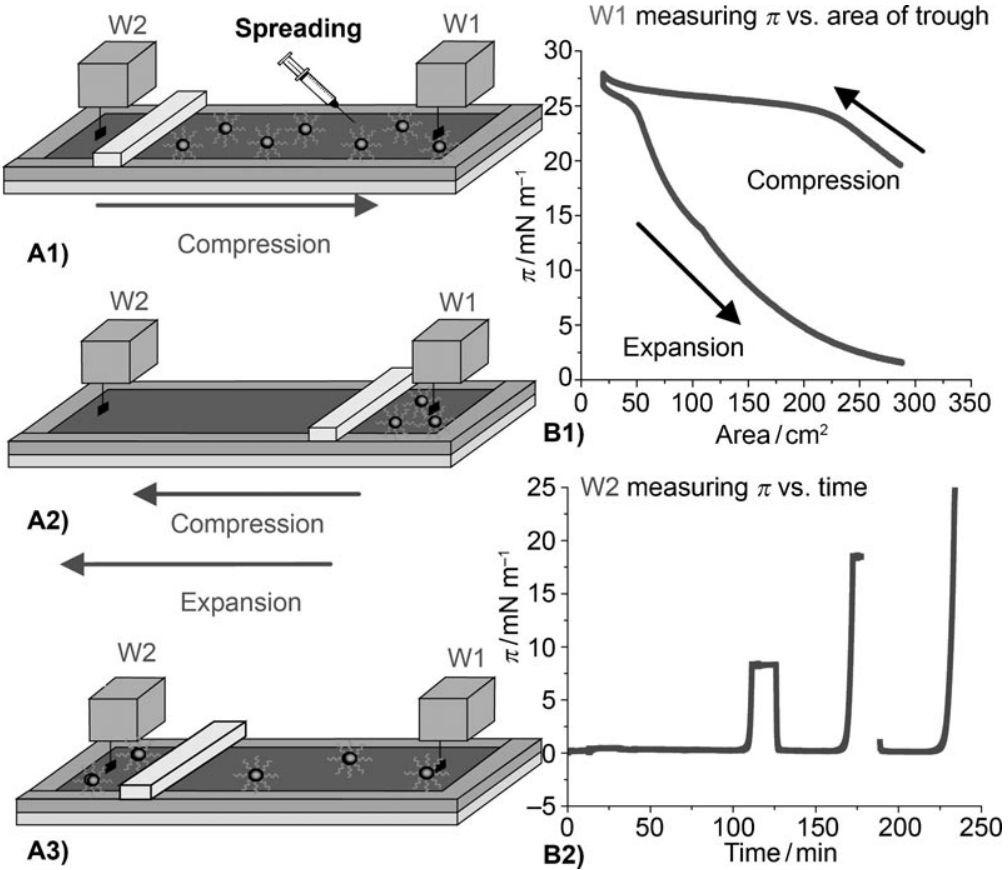

Figure 3. A) Schematic representation of the setup and the procedure used. B) First compression-expansion isotherm of the NPs (B1) obtained on the spreading area and the variation of the surface pressure in time (B2) on the left-hand side of the barrier. Three compression-expansion cycles are shown (B2). layer to pressures above the critical pressure leads to the desorption of the NPs from the interface (right-hand side of the barrier) into the subphase. The desorbed NPs adsorb slowly at the surface on the left compartment, which offers a large unoccupied surface area. The desorption/readsorption process was further confirmed by atomic force microscopy (AFM) measurements of samples prepared by the Langmuir-Schaefer technique (Figure 4A) from the left side of the barrier. Figure $4 B$ shows an AFM image obtained for the NP layer transferred at a surface pressure of $23.5 \mathrm{mN} \mathrm{m}^{-1}$ onto freshly prepared mica. The image proves the presence of the $\quad \mathrm{Fe}_{3} \mathrm{O}_{4} @ \mathrm{MEO}_{2} \mathrm{MA}_{90}$-co$\mathrm{OEGMA}_{10}$ NPs at the interface (on the left-hand side of the barrier) and the same lack of dense and ordered packing of the met-

formed only on the right-hand side of the one-barrier Langmuir trough (Figure $3 \mathrm{~A} 1$ ) by spreading. By using two surfacepressure microbalances (W1 and W2), we were able to meaallic cores as on the spreading compartment (transmission electron microscopy, TEM, image in Figure 4C). Moreover, the enrichment of the left compartment with the NPs was proved sure, at the same time, the sur-

face pressure on both sides of the barrier. Compression and expansion of this film led to the already discussed pressure/area isotherm (Figure 3B1). The NP film was kept in the compressed state above $\pi_{c}$ for more than one hour (Figure $3 \mathrm{~A} 2$ ). No variation of the surface pressure was recorded during this time on either side of the barrier. However, after compression towards the left-hand side of the trough (expansion of the right side, Figure $3 \mathrm{~A} 3$ ), an increase in the surface pressure on the left-hand side (and a decrease on the spreading area) was measured. After spreading new NPs and repeating the above-described experiment two more times, an increase of the surface pressure up to a value of $23 \mathrm{mN} \mathrm{m}^{-1}$ could be recorded on the left side of the barrier (Figure 3B2).

The results obtained suggest that the compression of the NP

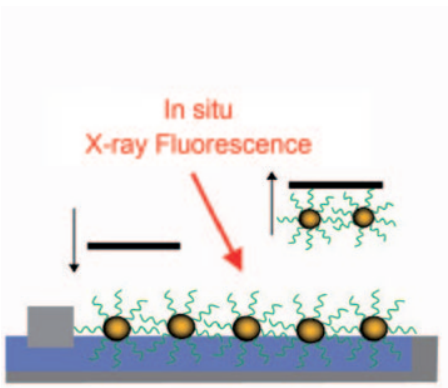

A) Langmuir-Schaefer transfer for AFM and TEM experiments
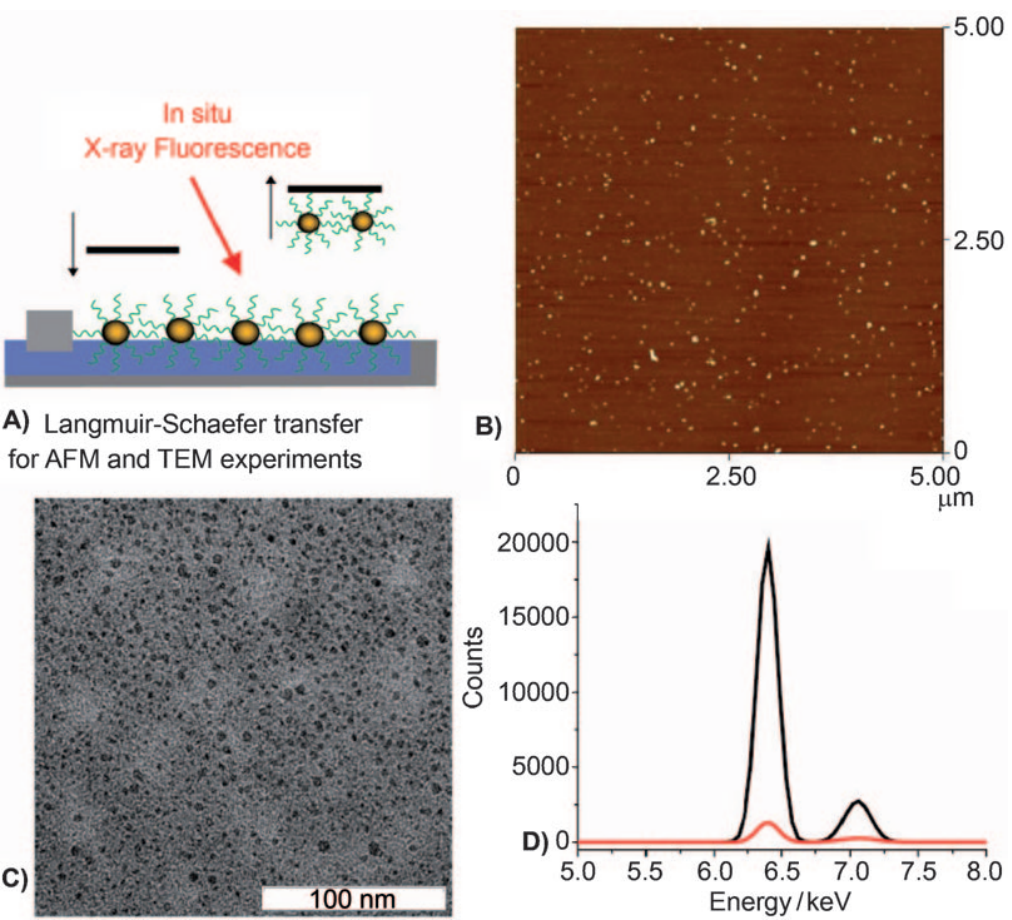

Figure 4. A) Schematic representation of the NP layer at the air/water interface. B) AFM and C) TEM images of the NP Langmuir layer, transferred on mica or on a copper grid, respectively, by the Langmuir-Schaefer technique. D) X-ray fluorescence spectra, taken at 0.6 (red) and $5.4 \mathrm{mN} \mathrm{m}^{-1}$ (black), showing the characteristic Fe $\mathrm{K} \alpha$ and $\mathrm{Fe}$ $\mathrm{K} \beta$ peaks. 
by measuring in situ X-ray fluorescence spectra at different surface pressures. Figure 4D shows the increase of the intensity of the characteristic Fe K $\alpha(6.4 \mathrm{keV})$ and $\mathrm{Fe} \mathrm{K} \beta(7.06 \mathrm{keV}) \mathrm{X}$-ray fluorescence peaks upon compression of the adsorption layer. The increase of the fluorescence intensity does not correlate in a linear manner with the compression ratio. This is an additional indication of a non-homogeneous distribution of the polymer-dressed NPs after re-adsorption, which is supported by the non-homogeneous NP number density seen in the AFM images.

These results indicate that the polymer and polymer-dressed NPs (after spreading from a chloroform solution) are trapped at the air/water interface. The film is even stable in contact with a polymer or NP-free subphase. The above-described configuration of the polymer at the interface, with a hydrophobic and a hydrophilic face, is obviously energetically more favorable for the stabilization of the polymer film than for desorption into the bulk. Compression of such a layer merely leads to the approach and rearrangement of the material. Only above the critical pressure do the polymer or the NPs desorb into the aqueous phase. Once in the aqueous subphase, due to the amphiphilicity of the polymer, the NPs will again adsorb at the free air/water interface.

The air/water interfacial properties of Langmuir and Gibbs layers of $\mathrm{Fe}_{3} \mathrm{O}_{4} @ \mathrm{MEO}_{2} \mathrm{MA}_{90}$-Co-OEGMA 10 NPs are clearly dictated by the unique properties of the used copolymer. Due to the inherent hydrophobic-hydrophilic properties of the copolymer-and despite its high solubility in water-the polymer and polymer-dressed NPs proved to be surface-active. Their surface activity is concentration- and time-dependent. However, this polymer and the corresponding NPs do not behave as water-soluble amphiphiles. The surprising and not-reportedbefore properties are that once adsorbed at the air/water interface, the NPs do not exchange with material in the bulk but they are trapped at this interface. This means that the polymer and all the NPs from the bulk adsorb at the interface until a maximum coverage is reached. Once formed-either by adsorption or by spreading on the water surface-the interfacial films composed of $\mathrm{Fe}_{3} \mathrm{O}_{4} @ \mathrm{MEO}_{2} \mathrm{MA}_{90}$ - $\mathrm{CO}-\mathrm{OEGMA} \mathrm{A}_{10} \mathrm{NPs}$ are energetically stabilized and kinetically long-time stable (up to a critical surface pressure of approximately $25 \mathrm{mN} \mathrm{m}^{-1}$ ) due to a special orientation of the polymer chains. Furthermore, we proved that the copolymer and the NPs are hydrophilic enough to be squeezed-out from the interface, if the packing density exceeds a critical value, and to be dispersed in the aqueous subphase without precipitation. Future work will be devoted to study their interfacial activity and partitioning into membrane models, for example, by variation of temperature, salt and copolymer composition. This is a very important aspect, considering our interest in studying and understanding the ability of these NPs to cross biological membranes.

\section{Acknowledgements}

We thank Dr. R. Miller for giving us the possibility to perform the dynamic surface tension measurements, Dr. V. Pradines for his help, A. Heilig for the AFM measurements, R. Pitschke for the TEM measurements and M. Meckelburg for the preparation of the NP colloidal dispersions. We thank HASYLAB at DESY, Hamburg, Germany, for beamtime and excellent support. This work was supported by the Max Planck Society.

Keywords: interfaces - Langmuir-Gibbs layers - magnetite nanoparticles $\cdot$ polymers

[1] A. K. Gupta, M. Gupta, Biomaterials 2005, 26, 3995-4021.

[2] N. Butoescu, C. A. Seemayer, G. Palmer, P. A. Guerne, O. Jordan, Arthritis Res. Ther. 2009, 11, 3.

[3] A. K. Gupta, A. S. G. Curtis, Biomaterials 2004, 25, 3029-3040.

[4] A. K. Gupta, M. Gupta, Biomaterials 2005, 26, 1565-1573.

[5] C. Corot, P. Robert, J.-M. Idée, M. Port, Advanced Drug Delivery Reviews 2006, 58, $1471-1504$

[6] M. F. Kircher, J. R. Allport, E. E. Graves, V. Love, R. Weissleder, Cancer Res. 2003, 63, 6838-6846

[7] H. Zeng, J. Li, J. P. Liu, Z. L. Wang, S. Sun, Nature 2002, 420, 395.

[8] E. M. Claesson, A. P. Philipse, Langmuir 2005, 21, 9412-9419.

[9] S. B. Bubenhofer, E. K. Athanassiou, R. N. Grass, F. M. Koehler, M. Rossier, W. J. Stark, Nanotechnology 2009, 20485302.

[10] M. Chanana, S. Jahn, R. Georgieva, J.-F. Lutz, H. Baumler, D. Wang, Chem. Mater. 2009, 21, 1906-1914.

[11] M. Paulus, P. Degen, S. Schmacke, M. Maas, R. Kahner, B. Struth, M. Tolan, H. Rehage, Eur. Phys. J. Special Topics. 2009, 167, 133-136.

[12] P. Degen, M. Paulus, M. Maas, R. Kahner, S. Schmacke, B. Struth, M. Tolan, H. Rehage, Langmuir 2008, 24, 12958-12962.

[13] D. K. Lee, Y. H. Kim, Y. S. Kang, P. Stroeve, J. Phys. Chem. B 2005, 109, $14939-14944$.

[14] F. C. Meldrum, N. A. Kotov, J. H. Fendler, J. Phys. Chem. 1994, 98, 4506.

[15] D. K. Lee, Y. H. Kim, C. W. Kim, H. G. Cha, Y. S. Kang, J. Phys. Chem. B 2007, 111, 9288-9293.

[16] H. Li, R. Sachsenhofer, W. H. Binder, T. Henze, T. Thurn-Albrecht, K. Busse, J. Kressler, Langmuir 2009, 25, 8320-8329.

[17] J.-F. Lutz, J. Polym. Sci. Part A Polym. Chem. 2008, 463459.

[18] R. L. Shuler, W. A. Zisman, J. Phys. Chem. 1970, 74.

[19] J. L. Zatz, J. Pharm. Sci. 1978, 67, 1464.

[20] B. B. Sauer, H. Yu, Macromolecules 1989, 22, 786-791.

[21] J. C. Scott, R. W. B. Stephens, The Journal of the Acoustical Society of America 1972, 52, $871-878$.

[22] J. Maldonado-Valderrama, M. J. Galvez-Ruiz, A. Martin-Rodriguez, M. A. Cabrerizo-Vilchez, Langmuir 2004, 20, 6093-6095.

[23] D. Cho, M. A. Cornec, Bull. Korean Chem. Soc. 1999, 20, 999-1004.

Received: September 23, 2010

Published online on November 5, 2010 